\title{
CCN Traffic Optimization for IoT
}

\author{
Jérôme François, Thibault Cholez, Thomas Engel \\ Interdisciplinary Centre for Security, Reliability \& Trust - University of Luxembourg, \{firstname.name\}@uni.lu
}

\begin{abstract}
Content-Centric Networking (CCN) recently received a lot of attention thanks to its elegant way to optimize content diffusion at the scale of Internet. However, communications occurring at the edge of Internet, in particular the Internet of Things (IoT), are also a vivid research topic. Even if CCN was not initially designed to optimize the specific traffic pattern of the IoT, it can be improved to better support these new applications. In this paper, we propose to optimize the traffic within a $\mathrm{CCN}$ for IoT network where information is created and consumed at different frequencies. The simulations show that our solution outperforms the vanilla $\mathrm{CCN}$ architecture for this generic scenario.
\end{abstract}

\section{INTRODUCTION}

Network protocols for the Internet-of-Things (IoT) are still a very active field of research. Even if 6LoWPAN/RPL [1] is currently well established, some disruptive research works [2], [3], [4] apply the new Information-Centric Networking (ICN) paradigm, designed to improve the core of the Internet, to other usages at the edge of the Internet, like the IoT. ICN is however not optimized by design for IoT communications which exhibit different properties (push-based, short-lived, small contents).

In this context, our approach optimizes the diffusion of information requested at different sampling rates and sent by sensors within the network using the Content-Centic Networking $(\mathrm{CCN})$ architecture. More precisely, we describe how a node in the path between a data producer and some consumers can maintain states to transmit the produced upstream data toward the downstream links with a limited overhead.

The rest of the paper is organized as follows: Section II presents the advantages of using CCN for the IoT. Section III presents our optimization reducing the number of messages as well as its evaluation through simulations. Finally, we present the related work in Section IV and conclude in Section V.

\section{CCN-BASED IOT}

\section{A. IoT requirements}

The IoT enables smart services through the interconnection of things having computational resources. Smart applications usually rely on contextual information like the geographical location or other physical sensors. Therefore, a core component of the IoT are the sensors or, in a more general way, "information producers". WSNs (Wireless Sensor Networks) are usually connected to the Internet through gateways [5]. The information may be produced in two different modes: either on demand when another entity requests it, or proactively sent to multiple subscribers.

Similarly to WSNs, the IoT undergoes scalability and low power efficiency challenges. For efficient information in WSNs, two major routing approaches [6] can be identified: data-centric and hierarchical protocols. Hierarchical approaches, as for example [7], try to cluster nodes and to elect a leader serving as a kind of proxy. Data-centric routing, like [8], advocates for a name based routing where data follows the reverse path of the corresponding query by a hop by hop mechanism.

We promote the usage of $\mathrm{CCN}$ for IoT due to existing implementations for computers and smartphones, CCNx [9], but also for sensors running Contiki [3]. Moreover, $\mathrm{CCN}$ combines the advantage of the data-oriented approach and hierarchical routing, by leveraging a scalable hierarchical naming scheme, and integrates security mechanisms and caching by design.

\section{B. $C C N$}

$\mathrm{CCN}$ is well suited for distributed environments where each node plays a role in the routing decisions. A node interested in a given content (consumer) sends an interest request which is forwarded until another node can satisfy it (producer) by sending a data message using the reverse path. Each CCN node maintains three vital types of information:

- $\quad$ the Pending Interest Table (PIT) tracks the forwarded interests not yet satisfied,

- the Forward Information Base (FIB) maintains the next hop information used to forward interests,

- the Content Store (CS) indexes the content which is actually stored or cached on the local node.

In $\mathrm{CCN}$, a face is anything serving as medium for transmitting and receiving messages. So, when a content $c$ is requested by an interest $i$ on a current node $n$, received from another node on the face $f$, the steps are as follows:

1) if $c$ is available locally ( $c$ is in the CS), $n$ sends it by a data message on the face $f$

2) if no match for $c$ exists in the CS but exists in the PIT, this means that $n$ already forwarded an interest for $c$. Hence, no message is emitted. If the new interest $i$ is coming from a new face $f$, the latter is added to the list of faces associated to $c$ in the PIT,

3) if no match exists in the PIT and in the CS, $i$ is forwarded to another node using the FIB except if no route exists towards $c$, and the corresponding entry in created in the PIT.

The data message sent by the content provider is forwarded back hop-by-hop using the different PIT entries on the path as they contain the faces from where this content was requested.

More precisely, CCN leverages a hierarchical naming scheme such that data can only "satisfy" 


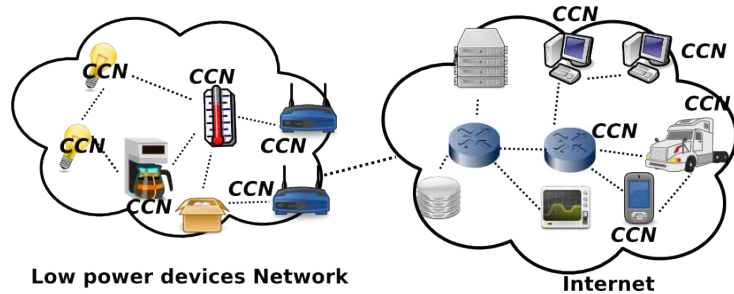

Fig. 1: A CCN enabled IoT Network

a specific interest whose content name is a prefix of the data message. For example, a content named ccnx:/uni.lu/videos/intro.avi defines several levels separated by /. To fill out the FIBs, nodes have to announce their content which is propagated to all other nodes with a limited overhead using aggregation. A typical example is the router of an organization which does not announce individual content but only a prefix like $c \mathrm{cnx}$ : /uni.lu.

\section{Problem definition}

A common IoT deployment is composed of communicating things in a dedicated network. Thanks to gateways, such a network is interconnected to the Internet. However, things tends also to be more and more directly connected, especially using cellular networks, like smartphones or connected cars.

We promote the use of $\mathrm{CCN}$ for low power devices. $\mathrm{CCN}$ considers every node as a router and so enhances direct neighbor to neighbor communications as in Figure 1. In addition, $\mathrm{CCN}$ is designed to be incrementally deployed beside IP which is necessary for traversing non-CCN paths.

However, this paper focuses on the low power devices at left hand in Figure 1. As highlighted before, $\mathrm{CCN}$ is well designed for distributed environments. Data-centric approaches are helpful for saving resources while the issue of a flat naming scheme is addressed with $\mathrm{CCN}$. In addition, the mechanisms of $\mathrm{CCN}$ can route interests in parallel on multiple paths and faces (and so communication medium) which thus helps to improve the QoS (opportunistic routing). Besides, the meaningful naming scheme enables new context-aware routing features[10].

These low power networks are composed of information producers (like sensors) requested by low power devices (like actuators) and other Internet devices (servers, smartphones, etc). Therefore, a single information producer may have several information consumers desiring different granularities. As an example, a temperature sensor can send information to a fire detection system at a high frequency, every 10 seconds, but such an information provided only every 5 minutes is enough for automatically adjusting a heating system.

Therefore, the information should not be multi-casted to every consumer at the same regularity to limit the communication overhead. Sending information temperature every 10 seconds during five minutes represents an overhead of 29 useless messages which can be forwarded through multiple paths and so consume even more resources.

The general scenario is depicted in Figure 2 where there is an intermediate $\mathrm{CCN}$ node $R_{i}$ which has to forward the information from $M$ producers transmitted by a previous router $R_{i-1}$ towards $K$ destinations which are accessible through other downstream nodes like $R_{i+1}$. The upstream and downstream routers can be also other producers or consumers as highlighted before. To reduce the number of messages forwarded by $R_{i}$, we consider a sub problem qualified as local regarding one router and one producer. Resolving the more general problem with multiple producers consists in resolving multiple times the previous problem.

Formally, the problem is defined as follows. Assuming (1) an intermediate node, a router $R_{i},(2)$ one producer $P$ able to send an information inf every $x$ seconds, (3) $\mathrm{N}$ consumers $C=c_{1}, \ldots, c_{N}$ and (4) that each consumer $c_{i}$ is interested to have inf every $x \times i_{i}$ seconds where $i_{i} \in \mathbb{N}$ (sampling period), the goal is to minimize the number of messages sent by $R_{i}$. Since each sensor node has a limited capacity, this optimization can only use a limited number of additional resources $\mathrm{Cap}_{i}$. This assumes that a proportion of resources is already reserved for the common $\mathrm{CCN}$ operations.

\section{SAMPLING OPTIMIZATION}

\section{A. Basic Forwarding strategies}

In the following discussions, the targeted router for sampling optimization purposes is $R_{i}$ and the considered producer is $P$. For sake of clarity, only one content is supposed for this producer, named $P_{\text {content }}$, but the approach can be easily applied in parallel to different contents.

By definition, $C C N$ nodes are stateful and only forward data on the back path if an interest was emitted beforehand. Therefore, a basic usage of $\mathrm{CCN}$ requires that information consumers directly request producers as illustrated in figure 3(a). This strategy is called pull and allows CCN to send data only when needed (on demand delivery) but the initial interest packet represents an overhead. However, an interest is not forwarded if a previous similar one has been already sent, and a given data message is sent only once per face which is then multi-casted by design. The producer also needs to initialize the FIB of the $\mathrm{CCN}$ nodes by announcing the hosted content. Such a step is not considered in the overhead computation because it is required only once.

Pushing data over CCN was envisioned in [2] which describes potential solutions and finally ends up designing a publish-subscribe mechanism where a node interesting in a certain content can subscribe for it, but in this case the diffusion is still based on IP. In this paper, we consider a strategy, push where data is directly transmitted using the FIB. In fact, the subscription is done in the same way that the registration of contents populating the FIB and the sensors' data dissemination is similar to the propagation of interests. In particular, sensors'data are not cached and corresponds to a one-way message. To do so, a simple option is to disseminate such very small and ephemeral data directly inside interests like for example / roomA/temperature/ts $=10 /$ value $=20$ assuming that the temperature sensor has registered the content /roomA/temperature/. Such an interest is forwarded using the FIB but without creating any entry in the PIT, since no data will be sent back. This can be easily done using a flag in the content name or in the interest header. However, a main advantage of creating an entry in the PIT, in the common usage 


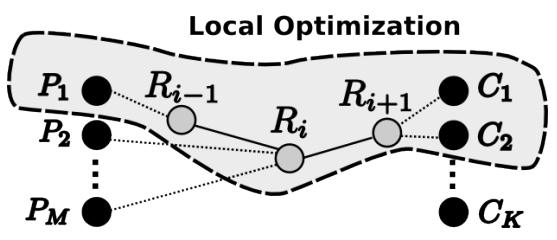

Fig. 2: Optimization problem

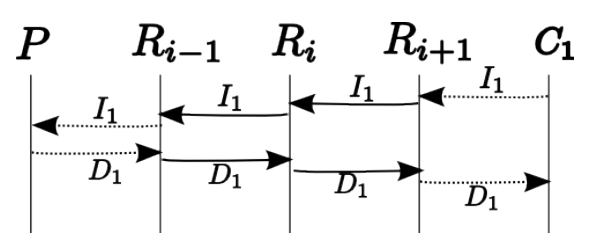

(a) On demand notification (pull)

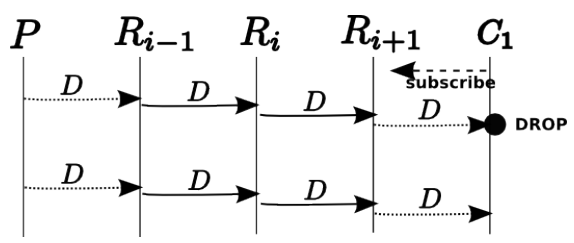

(b) Subscription based mechanism (push) - similar to IP multicast

Fig. 3: Basic Forwarding scheme (I: interest, D: data) (Dotted lines represents possible multiple hops)

of $\mathrm{CCN}$, is to avoid routing loops. Unlike [2] that uses standard IP unicast for avoiding routing loop, we propose to use a timestamp, $t s$, in the content name in conjunction with a new field in the FIB called last_seen in order to route only the latest content, which is the most valuable. Every new transmission for a given content will be checked against the last_seen value. More recent data will be forwarded while older obsolete ones will be discarded, thus avoiding loops and saving bandwidth.

Hence, we assume our aforementioned mechanism. This corresponds to data messages forwarded in Figure 3(b). However, since the information is transmitted regularly and independently of the demands, each value out of the sampling period $\left(i_{i}\right)$ of a consumer $c_{i}$ is dropped and represents an overhead of useless messages. Such a mechanism is comparable to use IP multi-cast where data is forwarded to all subscribers.

\section{B. Optimal Forwarding strategy}

To reduce the message overhead in an optimal way, the goal is to combine the advantage of both pulling and pushing modes. The router should only forward data to $C_{i}$ in pushing mode at the period $t$ only if $C_{i}$ has expressed its interest regarding this period: $t$ is a multiple of the sampling period $i_{i}$. Hence, when the consumers subscribe, they have to specify their sampling period such that $R_{i}$ can keep track of it thanks to a dedicated counter. This is shown in Figure 4(a) where $C_{1}$ subscribes to $P_{\text {name }}$ whit $i_{1}=2$. As highlighted, the counter is directly inserted in the FIB and updated each time a message of the subscribed data arrives.
However, this optimal strategy needs one counter per subscription. The additional resources $C a p_{i}$ are considered in terms of number of available counters, independently of the implementation. To limit the number of messages, identical messages are combined into one. Assuming $i_{1}=2$ and $i_{2}=3$, the sixth produced message is not forwarded twice as $D_{1}$ and $D_{2}$ but only once as $D_{1+2}$ meaning that this data serves both $C_{1}$ and $C_{2}$. The different notations of the same message just help in identifying the purpose of the message. In fact, all these messages are exactly the same and will be forwarded to both consumers thanks to the propagation mechanism of CCN, which makes feasible the use of a single message $D_{1+2}$. This simple mechanism is also applied to the other strategies described in the next section.

The optimal strategy (one counter per content per consumer) cannot be applied with a fixed $\operatorname{Cap}_{i}$ value because the number of subscriptions can be very large and the sensors' resources are very limited. While not being practical, this strategy is considered as the baseline for evaluating the performances of the other ones.

\section{Smart Forwarding strategies}

Assumes a fixed maximal number of counters $\mathrm{Cap}_{i}$, the goal of a smart forwarding strategy is to refine the pushing mode while limiting the number of forwarded messages. The worst case considers a single counter which serves to all subscribed periods. For example, with multiples like $i_{1}=2$ and $i_{2}=4$, a unique counter looping between 1 and 2 is

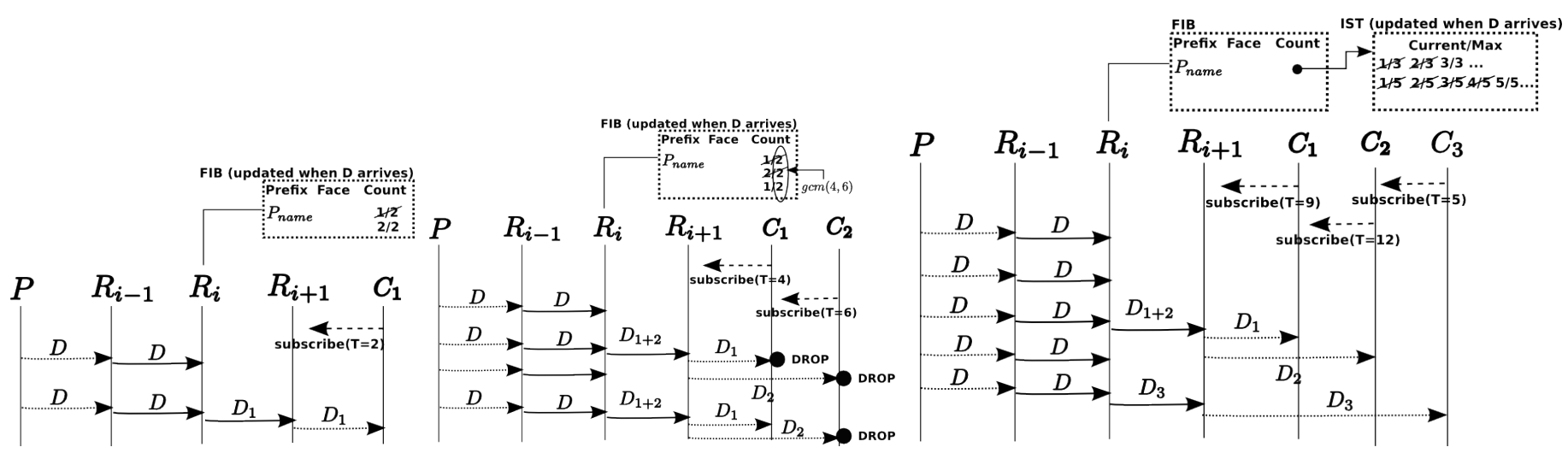

(a) Simple filtering

(b) GCD-based optimisation

(c) Using multiple counters in IST

Fig. 4: Sampling optimization (Dotted lines represents possible multiple hops) 
enough and does not entail additional messages. This can be extended using the Greatest Common Divisor (GCD) (strategy gcd). With $i_{1}=4$ and $i_{2}=6$ as in Figure 4(b), the unique counter loops over $\operatorname{gcd}(4,6)=2$ which thus avoids to forward message at every period. However, there is an overhead of useless messages like the first forwarded message $D_{1+2}$ which is then transferred by other routers as $D_{1}$ or $D_{2}$ before being dropped by the consumers. Moreover, there is a high risk that with numerous subscriptions, the global GCD reaches 1 which so does not represent any improvement compared to the basic pushing forwarding scheme.

With more counters, i.e. $\operatorname{Cap}_{i}>1$, this can be extended by selecting $C a p_{i}$ values, $G=\left\{g_{1}, \ldots, g_{C a p_{i}}\right\}$, such that:

$$
\forall C_{i} \in C, \exists g_{k} \in G, \alpha \in \mathbb{N}, g_{k} \times \alpha=i_{i}
$$

Figure 4(c) considers two counters and so introduces a specific structure, the Information Sampling Table (IST), which keeps track of them. With three consumers and $i_{1}=9, i_{2}=12$ and $i_{3}=5, R_{i}$ will forward every 3 and 5 data messages. Again, this will entail some message overhead but it is better than having considered the unique GCD which is one.

After having filtered identical intervals and those being multiple of others, the smart forwarding strategy affects one counter per unique remaining sampling interval $G^{\prime}=$ $\left\{g_{1}, \ldots, g_{M}\right\}$. If the number of needed counters is acceptable, i.e. $\left|G^{\prime}\right| \leq C_{a p}$, the algorithm stops and $G=G^{\prime}$. Otherwise it iterates until this condition is reached by merging at each step two counters, $g \_$merge $=\operatorname{gcd}\left(g_{i}, g_{j}\right),\left(g_{i}, g_{j}\right) \in G^{\prime} \times G^{\prime}, g_{i} \neq$ $g_{j}$. These counters are selected to maximize the GCD in order to limit the message overhead:

$\forall\left(g_{k}, g_{l}\right) \in G^{\prime} \times G^{\prime},\left(g_{k}, g_{l}\right) \neq\left(g_{i}, g_{j}\right), \operatorname{gcd}\left(g_{j}, g_{i}\right) \geq \operatorname{gcd}\left(g_{k}, g_{l}\right)$

\section{Evaluation}

For evaluation purposes, a dedicated simulator has been developed which implements the only necessary functions of $\mathrm{CCN}$ which are used in our approach. The number $m s g_{s}$ of messages forwarded by $R_{i}$ is considered regarding the chosen strategy $s$ (among pull, push, optimal, gcd or smart) and a configuration defined by:

- $\quad M A X \_T$ : the maximal length of the sampling interval that a consumer can subscribe to,

- $\quad N=|C|$ : the number of consumers which subscribes to a unique sampling interval. The latter is chosen randomly between 1 and $M A X_{-} T$,

- \#counters: the number of available counters (only impacts the smart forwarding strategy),

- \#steps is the number of simulation steps assuming that the length of a step is defined by the initial sampling period at which the sensor can produce data.

We measured the number of messages because it is clearly correlated to the energy consumption which is one of the main criteria for designing protocols for WSNs. Based on preliminary experiments, \#steps was fixed to $5 \times M A X \_T$, which guarantees that every consumer has received data at least five times during the discrete events simulation. As previously
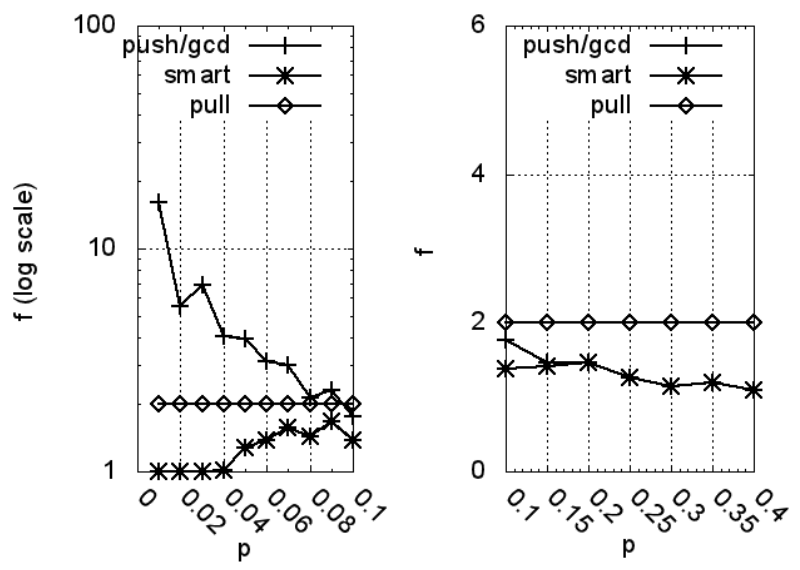

(a) Impact of the number of consumers $\left(M A X_{-} T=3000\right)$

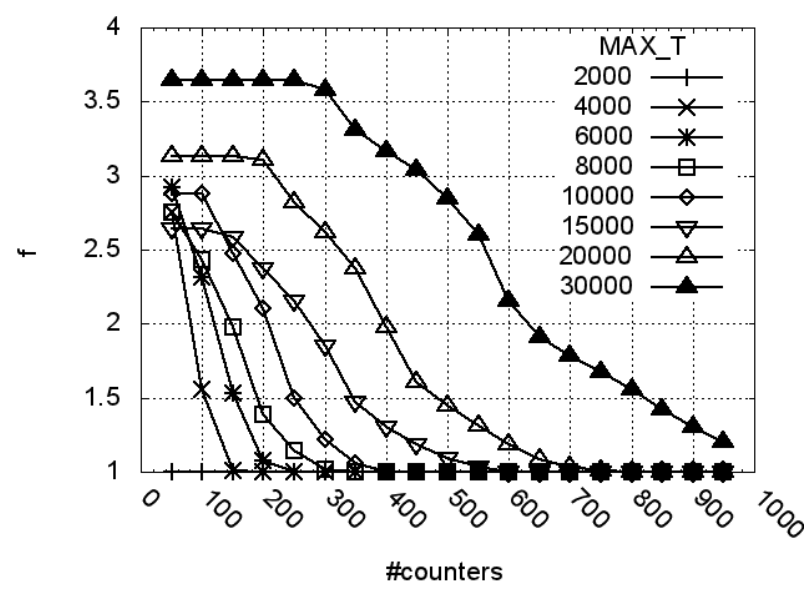

(b) Impact of the number of counters (smart forwarding, $p=0.05 \times$ $M A X_{-} T$ )

Fig. 5: Message overhead evaluation

said, the optimal strategy is used as the baseline to compute an overhead ratio defined as:

$$
f_{s}=\frac{m s g_{s}}{m s g_{\text {optimal }}}
$$

We always calculate the average of $f$ over 10 experiments. The number of consumers affects our performances by increasing the number of different sampling periods and is therefore expressed as a proportion $p$ of $M A X_{-} T$.

The number of consumers is evaluated in figure 5(a) where $M A X \_T=3000$ and \#counters $=100$. The vanilla $\mathrm{CCN}$ architecture (pull) highlights a ratio of 2 which is coherent since every data is sent after an initial interest. When $p$ increases, all other curves tend to one because the optimal strategy also requires to forward more messages as there are more customers. In particular, when $p$ increases, the chance to have a consumer $C_{i}$ having subscribed with an interval $i_{i}=1$ is higher which thus leads to forward a message at every step. Due to the same mechanism, the benefit of smart forwarding compare to the push mode is reduced. Therefore, using optimization has logically a higher impact when the number of subscribed intervals is low. However, the worst case for smart routing is for $p=0.08$ representing 240 
distinct intervals which are handled by the 100 counters. As highlighted, the $g c d$ strategy leads to the same result that the simple push strategy which forwards every data message because the overall GCD computation reaches always 1 even with $p=0.05$ due to the probability to have randomly selected one of the 430 prime numbers between 1 and 3000. Besides, as previously highlighted, the push mechanism is similar to IP multicast, which thus allows to compare our approach with the latter.

The smart forwarding strategy proposed in this paper always exhibits a ratio significantly lower than the classical $\mathrm{CCN}$ approach. It varies between 1 and 1.67. When $p$ is very small, there are enough counters to be optimal and starting at $p=4 \%$, the optimization starts by sharing the counters for multiple intervals which entails a message overhead. Around 0.1 , the optimal strategy has to forward many messages which thus highly impacts on the denominator in equation (1).

In figure 5(b), the number of counters for the smart forwarding strategy varies on $\mathrm{x}$-axis and different maximal sampling intervals have been tested. The number of uniquely subscribed intervals is set to $5 \%$ of $M A X_{-} T$ and so varies between 100 and 1500 depending on the configuration. When the number of usable counter increases, the overhead is logically reduced, even faster if there are less subscribed intervals (lower $\left.M A X \_T\right)$. Such an experiment helps to choose the right tradeoff between message overhead and hardware/software additional resources (number of counters). Then, depending on the communication medium and the hardware, it would be possible possible to select the most efficient number of counters in terms of energy consumption. For instance, assuming that the maximal acceptable overhead is expressed as $f=2$, the proper number of counters can be chosen regarding the number of uniquely subscribed intervals.

\section{RELATED WORK}

As mentioned, an important missing feature to use $\mathrm{CCN}$ for IoT is a push-based mechanism for communications. Instead of probing a sensor regularly, which consumes energy, a better way would be to let the sensor transmit its data when needed. Doing this in CCN is not natural but yet feasible. Van Jabobson et al. solved a similar issue to enable CCNbased conversational communications for voice over IP [11]. They propose a solution based on two properties of $\mathrm{CCN}$ : on-demand publishing to request content that has not been published yet, which simulates a rendez-vous point, and a deterministic algorithm followed by the two parties to find this service name. The approach in [2] handles small ephemeral messages, more precisely events communication typically produced by IoT. This allows CCN to support publish/subscribe communications by adding a new unicast forwarding table and a new set of dedicated messages: "one way" messages for event notifications that can follow the path of subscriptions up to the subscribers. In [3], a full CCN layer is provided for Contiki which enables CCN usage for low power devices and IoT.

Our paper also deals with the problem of efficient dissemination of event notifications in a sensor network. Some papers are close to this topic even if they do not optimize the diffusion of information according to fixed sampling rates. For example, the authors in [12] used non-linear optimization theory to optimize the rate at which sensors can be probed by a control system under network limitations. Another way to save network resources in WSNs is to rely on in-network aggregation techniques which have been largely investigated through different strategies [13], even secured ones [14]. This solution is particularly efficient when data is gathered by a few sinks while we envision in our case a more neighbor-toneighbor communication pattern between sensors.

\section{CONCLUSION}

In this paper, $\mathrm{CCN}$ is leveraged for distributed IoT communications, especially in the context of producers regularly updating information sent to multiple consumers. A new pushbased mechanism was designed and optimized. Even under strict resource constraints, the nodes optimize the forwarding of messages based on the update frequencies required by the different consumers. Compared to the optimal but unrealistic approach, the results show a limited overhead, always under the regular CCN pull approach. Our future work will focus on improving our approach for multiple-content producers.

Acknowledgement: This work was partly funded by BUTLER and IoT6 FP7 EU projects under the grant agreements 287901 and 288445.

\section{REFERENCES}

[1] G. Montenegro, N. Kushalnagar, J. Hui, and D. Culler, "Transmission of ipv6 packets over ieee 802.15.4 networks," 2007. [Online]. Available: http://tools.ietf.org/html/rfc4944

[2] A. Carzaniga, M. Papalini, and A. L. Wolf, "Content-based publish/subscribe networking and information-centric networking," in $A C M$ SIGCOMM workshop on Information-centric networking - ICN, 2011.

[3] B. Saadallah, A. Lahmadi, and O. Festor, "CCNx for Contiki: implementation details," INRIA, Tech. Report RT-0432, 2012.

[4] S. Y. Oh, D. Lau, and M. Gerla, "Content centric networking in tactical and emergency MANETs," in Wireless Days (WD), 2010 IFIP. IEEE, Oct. 2010.

[5] L. Steenkamp, S. Kaplan, and R. Wilkinson, in AFRICON, 2009.

[6] A. Boukerche, M. Z. Ahmad, D. Turgut, and B. Turgut, A Taxonomy of Routing Protocols in Sensor Networks. John Wiley \& Sons, Inc., 2008, pp. 129-160.

[7] S. Soro and W. B. Heinzelman, "Cluster head election techniques for coverage preservation in wireless sensor networks," Ad Hoc Netw., vol. 7, no. 5, pp. 955-972, Jul. 2009.

[8] C. Intanagonwiwat, R. Govindan, and D. Estrin, "Directed diffusion: a scalable and robust communication paradigm for sensor networks," in Conference on Mobile computing and networking. ACM, 2000.

[9] Content Centric Networking. [Online]. Available: http://www.ccnx.org

[10] V. Jacobson, D. K. Smetters, J. D. Thornton, M. F. Plass, N. H. Briggs, and R. L. Braynard, "Networking named content," in International conference on Emerging networking experiments and technologies CoNEXT. ACM, 2009.

[11] V. Jacobson, D. K. Smetters, N. H. Briggs, M. F. Plass, P. Stewart, J. D. Thornton, and R. L. Braynard, "VoCCN: voice-over content-centric networks," in Workshop on Re-architecting the internet - ReArch. ACM, 2009.

[12] J. Mao, Z. Wu, X. Wu, and S. Wang, "Sampling frequency optimization in wireless sensor network-based control system," in International conference on Advanced Web and Network Technologies, and Applications - APWeb. Springer, 2006.

[13] E. Fasolo, M. Rossi, J. Widmer, and M. Zorzi, "In-network aggregation techniques for wireless sensor networks: a survey," Wireless Communications, IEEE, vol. 14, no. 2, pp. 70-87, 2007.

[14] L. Yu, J. Li, S. Cheng, and S. Xiong, "Secure continuous aggregation via sampling-based verification in wireless sensor networks," in INFOCOM, 2011 Proceedings IEEE, 2011, pp. 1763-1771. 